\title{
Los “One minute paper” como elemento de evaluación continua en la búsqueda de la mejora de la evaluación en una asignatura del Grado en Derecho.
}

\section{Ixusko Ordeñana Gezuraga}

Profesor Derecho Procesal Universidad del País Vasco, ixusko.ordenana@ehu.eus

\begin{abstract}
In the framework of new active teaching-learning methodologies, having recovered student's centrality in this process and adopting teacher's guidance task, evaluation formula becomes an essential element: it is essential to assess adequately that student acquires competences and knowledge linked to specific subject. Leaving aside old formulas that based student's evaluation exclusively on a final exam, an experience of teaching innovation developed in a subject of Law Degree, in the first semester of 2017/2018 academic year, is described: 5 one minute paper have been combined with one final test, using a formative and summative evaluation formula.
\end{abstract}

\section{Keywords:}

One minute paper, evaluation, improving, teaching-learning process

\section{Resumen}

En el marco de las nuevas metodologías activas de enseñanza-aprendizaje, habiendo recuperado el estudiante la centralidad que le corresponde en este proceso y adoptando el docente la labor de guía, deviene elemento esencial la fórmula de evaluación: es imprescindible tasar adecuadamente que el alumnado adquiere las competencias y conocimientos vinculados a la asignatura concreta. Prescindiendo de formulas antiguas que basaban la evaluación exclusivamente en un examen final, se presenta la experiencia de innovación docente desarrollada en el primer cuatrimestre del curso 2017/2018 en una asignatura del Grado en Derecho: se han combinado 5 “one minute paper” con una prueba final, utilizando una fórmula de evaluación formativa y sumativa.

\section{Palabras clave:}

“One minute paper”, evaluación, mejora, proceso enseñanza-aprendizaje. 


\section{Introducción}

Seis años después de que la Universidad del País Vasco-Euskal Herriko Unibertsitatea ${ }^{1}$ introdujera el nuevo plan de estudios en la formación jurídica, familiarizados con las nuevas metodologías de enseñanza-aprendizaje, actuando en nuestra labor docente, conforme al aprendizaje cooperativo, aprendizaje basado en problemas y al método del caso, seguimos buscando la forma más adecuada de evaluar al alumnado. En este sentido, nos encontramos con un límite principal de orden público: la UPV-EHU, en general, y su Facultad de Derecho, en particular, nos exigen que, como mínimo, el alumno pueda obtener 3 puntos sobre 10 a lo largo del curso, mediante pruebas, ejercicios o prácticas varias. En este contexto, los últimos cursos hemos seguido distintas fórmulas de evaluación, si bien a todas les hemos detectado defectos o taras. Estabamos intentando buscar la fórmula "cuasiperfecta", cuando en un curso de formación docente ofrecido por el Servicio de Asesoramiento Educativo de la UPV/EHU, nos hablaron del "One minute paper" como fórmula o prueba de evaluación continua. Convencidos de que nada perdíamos por probarlo -sumaría un intento más-, el primer cuatrimestre de este curso 2017/2018 lo hemos utilizado en tres grupos del Grado en Derecho como elemento de evaluación continua. En las siguientes líneas, queremos explicar la experiencia, calificándola, desde un principio, como éxito rotundo.

\section{Objetivos}

La experiencia que vamos a relatar en el IV Congreso Nacional de Innovacion Educativa y Docencia en red $^{2}$ persigue:

1. Partiendo de la importancia del método de evaluación del estudiantado en el marco de los sistemas activos de enseñanza-aprendizaje, fijar uno que case adecuadamente con las competencias a adquirir en la asignatura Derecho Procesal I, argumentando pedagógicamente la opción por el mismo.

2. Analizar el marco jurídico en el que nos podemos mover (la normativa de la UPV/EHU al respecto) y el margen de maniobra y mejora.

3. Exponer la implementación del método de evaluación empleado el primer cuatrimestre del curso 2017/2018, detectando sus bondades y debilidades. Se tendrá en cuenta, al efecto, especialmente la visión o perspectiva del alumnado, además de la del docente.

4. Obtener un feed-back por parte de los asistentes al IN-RED 2018, dirigido, en su caso, a mejorar el método de evaluación expuesto.

${ }^{1}$ En los sucesivo, UPV-EHU.

2En lo sucesivo, IN-RED 2018.

(c) EY-NC-ND 2018, Universitat Politècnica de València

Congreso IN-RED (2018) 


\section{Desarrollo de la innovación}

\subsection{Marco teórico de la experiencia de innovación: la evaluación universitaria y su importancia. Fundamentos pedagógicos y normativa vigente en la Universidad del País Vasco.}

\subsubsection{Líneas elementales de la evaluación universitaria hoy.}

En un contexto de educación superior, en el desarrollo de cualquier Grado universitario, deviene esencial tasar o valorar el aprendizaje del estudiantado ${ }^{3}$. No únicamente por resultar un elemento formal esencial, exigido institucionalmente para poder conceder un título oficial profesionalizante, sino -principalmente- por ser parte sustancial del proceso de enseñanza-aprendizaje. La evaluación es, en sí misma, un elemento de aprendizaje para el alumnado $^{4}$. No obstante, en la evaluación intervienen activamente los/as dos protagonistas: el/la docente y el/la alumno/a. Mediante la evaluación el profesor/a -encargado/a de plantear situaciones y ofrecer al alumnado los instrumentos para aprender-, persigue (1) hacerse con evidencias o pruebas procedentes de aquéllos/as, a los que (2) aplica unos criterios de corrección, que le permiten (3) comprobar el nivel de dominio de los objetivos planteados para la materia para, con ello, realizar un juicio de valor ${ }^{5}$. Los tres elementos integran lo que se denomina "sistema de evaluación".

Conforme a los nuevos tiempos y metodologías activas, concebido el aprendizaje "como proceso constructivo y no meramente receptivo" 6 , el sistema de evaluación no lo debe protagonizar el/la docente en su labor de calcular u obtener una nota final oficial que expresa un rendimiento académico. La evaluación debe centrarse especialmente en el estudiante, sirviendo para orientarle en su proceso de aprendizaje, motivarle y ayudarle a superar trabas. La evaluación ayuda especialmente al estudiante a conocer si su organización respecto a la materia (el tiempo y esfuerzo invertido y método utilizado) es adecuada y, por ende, y como consecuencia, a articular estrategias de futuro respecto al proceso de aprendizaje que protagoniza ${ }^{7}$. En este sentido, deviene esencial el feed-back o

\footnotetext{
${ }^{3}$ Describen la evaluación como parte esencial del proceso de enseñanza y aprendizaje, ÁLVAREZ VALDIVIA, I.M., ARTILES ARMADA, K. "La evaluación en la universidad: Estudio preliminar”, Aula Abierta (2001), núm. 78. Oviedo: Instituto de Ciencias de la Educación de la Universidad de Oviedo. En la misma línea, remarcando que es uno de los elementos que "mayor impacto" tiene en el estudiantado en su proceso de aprendizaje, GARGALLO CASTEL, A., "Aproximación al estudio de la evaluación en la universidad: un análisis exploratorio con alumnos semipresenciales”, Contextos educativos: Revista de educación (2009), núm. 12. La Rioja: Universidad de La Rioja.

4 En este último sentido, se habla de la "evaluación orientada al aprendizaje”. Explican su fundamento, incidiendo en sus tres elementos esenciales (participación activa de los estudiantes, retroalimentación y tareas auténticas), IBARRA SAIZ, M.S., RODRÍGUEZ GÓMEZ, G., GÓMEZ RUÍZ, M.A., "La evaluación entre iguales: beneficios y estrategias para su práctica en la universidad”, Revista de educación (2012), núm. 359. Madrid: Ministerio de Educación, Cultura y Deporte.

${ }^{5}$ Remarca que la evaluación siempre "aboca a un juicio sobre algo", PIDONE, C.L., "Evaluación de los aprendizajes en la Universidad”, Diálogos educativos (2005), núm. 9. Chile: Universidad Metropolitana de Ciencias de la Educación.

${ }^{6}$ Con SAENZ DEL BURGO MARTíNEZ, L., PURAS OCHOA, G., “Aplicación de metodologías activas para aprender a gestionar la oficina de farmacia: Una experiencia en el aula”, IJERI: International journal of Educational Research and Innovation (2018), núm. 9. Sevilla: Universidad Pablo de Olabide.

${ }^{7}$ En esta senda, apoyado en literatura del campo de la pedagogía, al tiempo que expone una experiencia de innovación docente en el ámbito jurídico, MORENO OLIVOS, T., “La evaluación del aprendizaje en la universidad: Tensiones, contradicciones y
} 
retroalimentación que aporta el/la docente al alumno/a ${ }^{8}$. Se aplaude, incluso, que el alumnado pueda elegir o configurar su propio sistema de evaluación ${ }^{9}$-algo por lo que hemos optado nosotros mediante el "contrato programa", como relataremos a posteriori-. Todo ello es parte fundamental de un aprendizaje significativo, en el que el alumno/a puede relacionar lo nuevo con lo que ya conoce ${ }^{10}$.

No se puede obviar, igualmente, que el método de evaluación es, al tiempo, un instrumento muy útil para el/la docente: le permite conocer las dificultades del estudiantado en la materia, y por tanto, donde hacer más hincapié en su impartición; es, al tiempo, un instrumento vital para la re-organización y planificación de la docencia (contenidos, formas en general,... $)^{11}$. Lo es porque muestra la congruencia entre los objetivos planteados en la guía docente para la asignatura y los resultados obtenidos.

El sistema de evaluación puede ser sumativo o formativo. El primero supone la emisión de un (único) juicio por parte del docente, al finalizar un período de enseñanza, para calificar en función del rendimiento apreciado. Es el mecanismo que denota que el/la estudiante posee las competencias que requieren el ejercicio profesional del Grado que estudia, en general, y que se vinculan con la disciplina o materia de la que es evaluado, en particular, y que se supone que antes de cursarla no tenía. El segundo, por su parte, supone la emisión, por parte del docente, de juicios a lo largo de un período de enseñanza, haciendo partícipe de ellos al alumnado, permitiendo esta práctica que ambos mejoren (optimicen, si se quiere, en términos empresariales) su rol en el proceso de enseñanza-aprendizaje ${ }^{12}$. Ambos sistemas no son, no obstante, incompatibles, entendiendo que es su conjunción lo que mejor casa con las nuevas metodologías activas y con la pedagogía constructivista.

Por último, convivene remarcar, como hemos apuntado, que en el contexto de las metodologías activas, evaluamos competencias que se muestran mediante tareas que recogen los resultados de aprendizaje correspondientes.

desafíos”, Revista mexicana de investigación educativa (2009), núm. 41. México: Consejo Mexicano de Investigación Educativa. También, JANÉ, M., “Evaluación del aprendizaje: ¿problema o herramienta?”, Revista de estudios sociales (2005), núm. 20. Colombia: Universidad de Los Andes-Facultad de Ciencias Sociales.

${ }^{8}$ Lo entienden "estrategia clave para mejorar la enseñanza y el aprendizaje”, CANO GARCÍA, E., CABRERA LANZO, N., “Evaluación formativa de competencias a través del blog en la Universidad”, Digital Education Review (2013), núm. 23. Barcelona: Universitat de Barcelona-Digital Education Observatory

9 En nuestro apoyo, PORTO CURRÁS, M., “Evaluación de estudiantes en la universidad de Santiago de Compostela: percepciones de los propios alumnos”, REDU: Revista de Docencia Universitaria (2009), núm. 3. Valencia: Red Estatal de Docencia Universitaria

${ }^{10}$ En este sentido, definen la evaluación como "evolución y cambio”, RAMOS HERNANDO, C., BERASALUCE DIÉZ, R., PEIRÓ I GREGORI, S. (2016), "La evaluación por competencias en la universidad”, AAVV, XIV Jornadas de redes de investigación en docencia universitaria, p. 2615: Alicante: Universidad de Alicante.

${ }^{11}$ Remarcan la importancia de la evaluación para el propio docente, atendiendo a su experiencia, QUIROGA, V., MENA GARCÍA, E., PORLAN JUY, J., MORALES MARTÍNEZ, E., MÁRQUEZ SÁNCHEZ, L., "La evaluación continua en el Grado de Trabajo Social de la Universidad de Barcelona”, Cuadernos de trabajo social (2014), núm. 2. Madrid: Universidad Complutense.

${ }^{12}$ Luego, el alumno puede mejorar el aprendizaje y el docente la enseñanza.

(c) B BY-NC-ND 2018, Universitat Politècnica de València

Congreso IN-RED (2018) 


\subsubsection{La normativa de evaluación vigente en la UPV/EHU}

Partiendo de la doble naturaleza de la evaluación (formal y pedagógica), el primer elemento nos dificulta modificaciones en el sistema de apreciación o control del aprendizaje que quebranten el marco mínimo garantizador que ordena la Universidad -en nuestro caso la UPV/EHU-. Concretamente, tenemos que estar al Acuerdo de 15 de diciembre de 2016, del Consejo de Gobierno de la UPV/EHU, por el que se aprueba la Normativa reguladora de la Evaluación del alumnado en las titulaciones oficiales de Grado $^{13}$. Resumimos sus líneas generales:

1.Se realiza una apuesta por los sistemas de evaluación continua, "facilitando y fomentando la implantación de sistemas de evaluación continua en los que el peso de pruebas finales se reduzca, e incluso desaparezca, a favor de la implantación de actividades de evaluación distribuidas a lo largo del periodo de docencia” (artículos ${ }^{14} 1$ y 8 )

2. Se reconoce el derecho del alumnado a ser evaluado con garantías de equidad y justicia, y de acuerdo con el nivel de enseñanza impartida (art. 2)

3. El objetivo de la evaluación es medir los resultados de aprendizaje "con los criterios e indicadores de evaluación específicos definidos en las guías docentes” (art. 4).

4. La evaluación debe ser formativa y ofrecer información y orientación adecuada al alumnado sobre el nivel de adquisición de competencias y las mejoras que se sugieren en su proceso de aprendizaje (art. 5)

5. Se preferencia la evaluación continua, quedando a salvo siempre el derecho del alumno "a ser evaluado mediante el sistema de evaluación final, independientemente de que haya participado o no en el sistema de evaluación continua" ${ }^{15}$ (art. 8.3)

6 En la evaluación continua, "las pruebas podrán realizarse exclusivamente a lo largo del periodo formativo o bien, realizarse a lo largo del periodo formativo, y complementarse con una prueba en la fecha oficial establecida para la convocatoria de exámenes correspondiente" (art. 8.2 a))

A priori, y conectando esta ordenación con la configuración de la evaluación en la universidad actual, vemos que la UPV/EHU acata los postulados y exigencias de los nuevos tiempos y nuevas metodologías de enseñanza-aprendizaje.

\subsection{Marco práctico de la experiencia de innovación o el cúmulo de problemas a tener en cuenta: los grupos a evaluar y el fracaso de métodos de evaluación anteriores.}

\footnotetext{
${ }^{13}$ Publicado en el Boletín Oficial del País Vasco del 13 de marzo de 2017.

${ }^{14}$ En lo sucesivo, art.

15 “Para ello, el alumnado deberá presentar por escrito al profesorado responsable de la asignatura la renuncia a la evaluación continua, para lo que dispondrán de un plazo de 9 semanas para las asignaturas cuatrimestrales y de 18 semanas para las anuales, a contar desde el comienzo del cuatrimestre o curso respectivamente, de acuerdo con el calendario académico del centro. La guía docente de la asignatura podrá establecer un plazo mayor” añade el precepto mentado.
} 


\subsubsection{El estudiantado a evaluar y sus circunstancias}

En el marco jurídico descrito, y para poder entender nuestra experiencia, deviene imprescindible examinar sus circunstancias. Conviene apuntar especialmente que el primer cuatrimestre del curso 2017/2018 hemos sido responsables de tres grupos de estudiantes ${ }^{16}$. Dos en el Grado en Derecho -uno de castellano y otro de euskera ${ }^{17}$ - y uno en el doble Grado Derecho y Administración de Empresas -también en euskera-. El grupo de castellano cuenta con 100 estudiantes y los de euskera con 40 más o menos cada uno. En suma sencilla, hemos enseñado a casi 200 alumnos y alumnas, de cuyas evaluaciones obviamente- también nos hemos encargado.

La asignatura que impartimos -y, por tanto, evaluamos- es Derecho Procesal I, dotada con una carga de 6 créditos. Se caracteriza por un temario muy extenso (15 lecciones) y esencial para la formación del futuro jurista, que se trabaja en el aula en 42 horas de clase magistral y 18 horas de prácticas. Conforme al cómputo del crédito europeo, estas actividades requieren, fuera del aula, 63 y 27 horas de trabajo del alumnado, respectivamente. Nuestra experiencia de más de 15 años en la impartición de la materia nos dicta que el estudiantado percibe la asignatura como de dificultad media-alta.

\subsubsection{Los sistemas de evaluación anteriores y sus circunstancias}

Con un contexto parecido al descrito, los últimos 6 cursos, el tiempo que lleva instaurado el Grado en Derecho en nuestra Facultad, y hasta dar con el método de evaluación implementado este curso 2017/2018, hemos utilizado previamente otros dos. Ambos tienen en común que valorábamos en 7 puntos la teoría de la materia y en 3 la práctica, cumpliendo la normativa de nuestra propia Facultad.

En uno primero, que implementamos durante tres cursos, el examen teórico era oral en la fecha oficial, y los puntos correspondientes a las prácticas se distribuían, para el alumnado de evaluación continua, en 3 prácticas que realizaban fuera del horario escolar y corregía el docente en su despacho individualmente y en el aula colectivamente. Estas prácticas eximían al estudiante, que optaba por la evaluación continua, de realizar un examen práctico (también valorado en 3 puntos) el día del examen oficial ${ }^{18}$. En juicio crítico de este sistema, y fijándonos únicamente en la evaluación continua -que, como hemos visto, es la que fomenta el marco normativo de la UPV/EHU-, el alumnado se quejaba de que las prácticas suponían mucha carga de trabajo y no tenía una repercusión similar en la nota final. Se alegaba mucho, asimismo, la dificultad de las prácticas, sugiriendo muchos estudiantes que sería mejor hacer mayor número de prácticas pero menos complejas. La prueba final -el examen oral, en el que el estudiantado debía responder a dos preguntas de otros tantos temas elegidos por sorteo-, que casaba muy bien con las competencias

\footnotetext{
${ }^{16}$ Describimos la situación de este último curso, sin perjuicio de que en los anteriores ha sido parecida, incluso, con mayor número de alumnado por grupo.

${ }^{17}$ Lenguaje cooficial de la Comunidad Autónoma vasca en la que se imparte docencia de Grado.

${ }^{18}$ Luego, a sensu contrario, el alumno/a que optaba por la evaluación final, tenía que realizar el día del examen oficial, una prueba oral teórica y otra práctica escrita.
}

(c)) EY-NC-ND 2018, Universitat Politècnica de València

Congreso IN-RED (2018) 
generales de la titulación y de nuestra asignatura (la comunicación es esencial, junto a la capacidad de síntesis y análisis crítico), tenía sus defensores y sus retractores en el estudiantado. Desde una perspectiva docente, las prácticas suponían una gran carga de trabajo, pues se corregían todas las prácticas individualmente y reportaba el correspondiente feed-back, colectivamente, en el aula e, individualmente, en el despacho. En relación a la prueba teórica oral nos preocupaba especialmente el grado de subjetividad en la evaluación, inevitable cuando se ha de hacer frente a 200 exámenes en pocos días consecutivos.

Valoradas las debilidades y fortalezas del sistema de evaluación descrito, escuchando especialmente las protestas y anhelos del alumnado, los siguientes dos cursos, modificamos el sistema de evaluación. Era nuestro segundo intento de mejora. El examen final dejó de ser oral y pasó a ser un test con 30 items, que se convertían en 7 puntos de la nota final. Con ello, entendíamos que ganábamos objetividad en la corrección, si bien perdíamos el trabajar con el estudiantado la competencia de comunicación oral, para ahondar en la de análisis crítico de la materia En cuanto a las prácticas, también valoradas en 3 puntos para la nota final para el alumando de evaluación continua, pasaron a ser 30 casos sencillos, que realizaban fuera del aula y se corregían colectivamente -todas- en el aula y 10 individualmente por el profesor. Los/as estudiantes que optaban por la evaluación final tenían que hacer una práctica valorada en 3 puntos el día del examen oficial. Entendemos que el gran acierto de este sistema fue el examen test. Nos garantizaba una corrección rápida, objetiva y un feed-back sencillo. Nos percatamos, al tiempo, que el índice de alumnos y alumnas que se presentaba al examen se incrementó de manera considerable. Tenemos que reconocer, asimismo, que el alumnado valora muy positivamente esta prueba. El tema de las prácticas nos siguió sin convencer: el estudiantado seguía reiterando la misma crítica (mucho trabajo para tan poco peso en la nota final) y nuestro trabajo aumentó considerablemente; si antes corregíamos 3 prácticas (aunque fueran complejas) a cada estudiante, ahora corregíamos 10. Nos pasamos semanas completas corrigiendo las prácticas.

\subsubsection{Conclusión: la necesidad de mejorar el sistema de evaluación.}

Entendiendo la evaluación como un elemento esencial de nuestra docencia ${ }^{19}$, y que no hay "una regla única para evaluar”20, aunque sí un marco normativo mínimo que hay que obedecer, buscando la mejora continua en el proceso de enseñanzaaprendizaje, para satisfacer, en primer lugar, al alumnado, y, en segundo, también al docente, el primer cuatrimestre del curso 2017/2018 hemos utilizado otra fórmula de evaluación. Relatamos, en lo sucesivo, la experiencia.

\footnotetext{
${ }^{19}$ Gráficamente, SANTOS GUERRA, M.A., “20 paradojas de la evaluación del alumnado en la universidad española”, Revista electrónica interuniversitaria de formación del profesorado (1999), núm. 1. Zaragoza: Asociación Universitaria de Formación del Profesorado-Universidad de Zaragoza, señala que se podría decir “dime cómo evaluas y te diré que tipo de profesional eres”.

${ }^{20}$ Literal, DÍAZ CANALS, J.F., MORATALLA ISASI, S., “Los exámenes en la Universidad”, Ensayos: Revista de la Facultad de Educación de Albacete (2009), núm. 24. Albacete: Universidad de Castilla-La Mancha: Escuela Universitaria de Magisterio de Albacete.
}

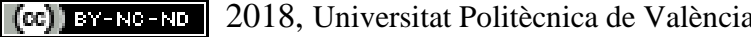




\subsection{El sistema de evaluación ideado y su implementación. El relato de una experiencia de innovación docente centrada en la evaluación continua.}

\subsubsection{Génesis del nuevo sistema de evaluación.}

Estábamos reflexionando sobre nuestro sistema de evaluación, sobre sus posibilidades de mejora, discutiendo propuestas con compañeros y compañeras de asignatura, cuando el Servicio de Asesoramiento Educativo de nuestra Universidad nos ofreció la posibilidad de asistir a un curso sobre evaluación en el marco de Formacion del Profesorado Universitario. En una formación excelente, se nos presentó un marco teórico y práctico que nos permitió conocer experiencias de otros centros y Grados. Nos tranquilizó sobremanera el constatar que el sistema de evaluación continua era un quebradero de cabeza para casi todos los docentes. ¿Cómo casar un trabajo equilibrado del alumnado con las exigencias de la materia? ¿Cómo ofecer un feed back adecuado al estudiantado? ¿Cómo evitar las horas interminables de corrección individual en el despacho? Eran cuestiones compartidas por profesores y profesoras asistentes.

De todas las fórmulas y posibilidades que se ofrecieron en el curso, entendimos que los “one minute paper" eran un instrumento que podía ayudarnos a mejorar el proceso de enseñanza-aprendizaje de nuestra materia, en general, y su sistema de evaluación, en particular.

\subsubsection{El "one minute paper" como recurso para mejorar la evaluación continua.}

Conforme a la literatura pedagógica ${ }^{21}$, el “one minute paper” (papel de un minuto, en español) es una técnica didáctica conforme a la cual, originalmente, al final de una clase magistral, el profesor/a pregunta a su aforo dos cuestiones: (1)¿Qué ha sido para tí lo más importante que has aprendido en esta clase? (2) ¿Qué es lo que te ha quedado más confuso? El alumnado debe responder brevemente, por escrito, en un minuto. Obtiene, así, el profesor un feed back claro y rápido, que le permite conocer la opinión del alumnado y preparar la próxima clase. Esta fórmula originaria, cuya utilización primaria se imputa a un profesor de Física de la Universidad californiana de Berkeley ${ }^{22}$, ha sido adaptada con éxito en muchas experiencias, manteniendo la esencia de fórmula consistente en "una tarea rápida por escrito" como base para un feed-back al tiempo que se ha convertido en

\footnotetext{
${ }^{21}$ La apunta, GIBBONS, R., “Adapting the One-Minute Paper for Active Learnig”, Academic Exchange Quarterly (2007), núm. 2. Chattanooga: Chattanooga State Technical Community College

${ }^{22}$ Por todos, ANGELO, T.A., CROSS, K.P. (1993), Classroom Assessment Techniques, 2 ed. San Francisco: Jossey Bass.
} 
un elemento de evaluación ${ }^{23}$. Las evidencias se pueden corregir fácil y rápidamente en el aula, guiados por el/la docente, trabajando sobre cada "paper" el propio alumno/a que lo ha confeccionado o un compañero/a suyo/a. También es posible que, primero, las corrija el docente fuera del aula y, luego, las lleve a ésta para comentarlas en grupo. Es indudable que, en cualquier caso, pueden funcionar como evidencia del domino de la materia por parte del estudiantado y que facilitan una retroalimentación rápida y sencilla.

\subsubsection{Sistema de evaluación propuesto: combinación de one minute paper y prueba objetiva final.}

Con la historia y antecedentes descritos, en las circunstancias apuntadas, respetando la normativa de evaluación vigente en la UPV/EHU, el primer cuatrimestre del curso 2017/2018 hemos implementado un sistema de evaluación diferente. Su primera característica es que se reconoce al alumnado el derecho de suscribir un contrato-programa para elegír la evaluación que quiere seguir en su proceso de aprendizaje: continua o final. La no suscripción del contrato se interpreta como opción por la final. Además, en su clausulado se recoge expresamente la posibilidad de abandonar el sistema de evaluación continua, debiendo notificarlo, como máximo, en la víspera del examen oficial ${ }^{24}$. Define el contrato-programa claramente ambos sistemas. "El sistema de evaluación continua: integrado por 5 pruebas parciales de estilo one minute paper (50\% calificación final) y un ejercicio téórico-práctico final de evaluación continua en forma de test, integrado por 30 items (50\% calificación final) ${ }^{25}$," "El segundo sistema: evaluación final: se integra de una única prueba test, que se realizará el día del examen oficial. Integrado por 40 items, de los que, al menos, 10 tendrán contenido meramente práctico, tiene un valor de 10/10 en la nota final". Centrándonos en el primero, se explica que "Siguiendo el método norteamericano conocido como one minute paper, durante el curso escolar, sin que sea necesaria la concreción de fecha con anterioridad, el alumnado deberá realizar 5 pruebas caracterizadas por su brevedad, que pueden consistir en: (1) respuesta a cuestión sobre lección trabajada por el alumnado fuera del aula, (2) respuesta a cuestión expuesta por el docente en el aula y (3) resolución de caso o cuestión práctica (expediente judicial, jurisprudencia, supuestos fácticos, escritos judiciales, tests,...) (...) Cada prueba/one minute paper tendrá un valor de 1/10 para la nota final; se corregirán en el aula y no es obligatoria la realización de todas ellas. El alumnado conocerá, con antelación y siempre antes del ejercicio final de evaluación continua, su resultado en las mismas".

\footnotetext{
${ }^{23}$ En este sentido, relatan como convirtieron las evidencias obtenidas mediante el "one minute paper” en elemento de evaluación continua, VIVEL BÚA, M., FERNÁNDEZ LÓPEZ, S., LADO-SESTAYO, R., “Innovación docente con One Minute Paper: ¿afecta al rendimiento académico?”, REDIE: Revista Electrónica de Investigación Educativa (2015), núm. 2. California: Universidad Autónoma de Baja California-Instituto de Investigación y Desarrollo Educativo.

${ }^{24}$ Se mejora, en este punto, la normative de la UPV/EHU a favor del alumnado.

${ }^{25}$ Nótese, pues, que la evaluacion continua suma la mitad de la nota final, lo que supone mejorar el porcentaje que exige la normativa de la Facultad (30\%), y cumple el deseo de la Universidad de preferenciar la evaluación continua.
} 


\section{Resultados}

Tras implementar el sistema de evaluación propuesto, fijándonos en la modalidad de evaluación continua basada en los ejercicios de one minute paper, podemos destacar los siguientes resultados:

1. Por primera vez, desde que se instauró el Grado en Derecho, y gracias a los que hemos llamado contrato-programa, el alumnado ha participado activamente en la elección de su sistema de evaluación, elemento eje del proceso enseñanzaaprendizaje, contribuyendo a que se sienta más protagonista de éste.

2. El $80 \%$ del alumnado ha optado por la evaluación continua ab initio, habiéndola abandonado únicamente el $2 \%$.

3. Los one minute paper han resultado un ejercicio excelente de evaluación continua. Desde la perspectiva docente, han resultado un instrumento ágil y sencillo para adquirir evidencias del nivel de conocimiento y dominio de la materia por parte del alumnado; han facilitado la retroalimentación y han reducido considerablemente la carga de tarea del docente. En opinión del estudiantado, es "la primeva vez que siguen una verdadera evaluación continua”, que les "ha exigido llevar la materia al día” y "les ha facilitado mucho su superación gracias a la retroalimentación del docente".

4. Los datos objetivos avalan la experiencia: los resultados finales han sido los mejores de los últimos años. No sólo ha sido superior el índice de superación de la materia (70\% del alumnado) sino, también, las calificaciones finales obtenidas (más de un $60 \%$ ha obtenido un notable).

\section{Conclusiones}

La experiencia de innovación docente implementada el primer cuatrimestre del curso 2017/2018, centrada en la mejora de la evaluación, en general, y de la evaluación continua, en particular, mediante los ejercicios denominados one minute paper, nos permite concluir:

1.Es posible y totalmente beneficioso para el proceso de enseñanza-aprendizaje implicar al alumnado en la forma de evaluación de éste. Funciona como elemento motivador e incentivador, no sólo del estudiante, sino también del docente.

2. Entendemos fundamental que el estudiantado aprehenda los beneficios y ventajas de la evaluación continua, sistema de evaluación que está fomentando la normativa universitaria y que casa perfectamente con las metodologías activas y el rol que éstas exigen al estudiante y al docente. Somos consciente, al tiempo, de la dificultad que supone para el docente implementar una verdadera y adecuada evaluación continua.

3. Después de muchos años de búsqueda e intentos más o menos acertados, el one minute paper ha resultado un instrumento muy pedagógico, eficiente y adecuado para desarrollar la evaluación continua. Especialmente destacables son el feed-back que facilita; la evidencia que constituye y las opciones de actuación posterior que permite.

(cc) EY-NC-ND 2018, Universitat Politècnica de València

Congreso IN-RED (2018) 
4. La experiencia de innovación relatada nos ha mostrado in situ, en el aula, con nuestro estudiantado, la trascendencia de la adecuada configuración e implementación del sistema de evaluación en el resultado final del proceso de enseñanza y aprendizaje.

\section{Referencias}

ÁLVAREZ VALDIVIA, I.M., ARTILES ARMADA, K. "La evaluación en la universidad: Estudio preliminar”, Aula Abierta (2001), núm. 78. Oviedo: Instituto de Ciencias de la Educación de la Universidad de Oviedo.

ANGELO, T.A., CROSS, K.P. (1993), Classroom Assessment Techniques, 2 ed. San Francisco: Jossey Bass.

CANO GARCÍA, E., CABRERA LANZO, N., "Evaluación formativa de competencias a través del blog en la Universidad”, Digital Education Review (2013), núm. 23. Barcelona: Universitat de Barcelona-Digital Education Observatory

DÍAZ CANALS, J.F., MORATALLA ISASI, S., "Los exámenes en la Universidad", Ensayos: Revista de la Facultad de Educación de Albacete (2009), núm. 24. Albacete: Universidad de Castilla-La Mancha: Escuela Universitaria de Magisterio de Albacete.

GARGALLO CASTEL, A., “Aproximación al estudio de la evaluación en la universidad: un análisis exploratorio con alumnos semipresenciales”, Contextos educativos: Revista de educación (2009), núm. 12. La Rioja: Universidad de La Rioja.

GIBBONS, R., “Adapting the One-Minute Paper for Active Learnig”, Academic Exchange Quarterly (2007), núm. 2. Chattanooga: Chattanooga State Technical Community College

IBARRA SAIZ, M.S., RODRÍGUEZ GÓMEZ, G., GÓMEZ RUÍZ, M.A., "La evaluación entre iguales: beneficios y estrategias para su práctica en la universidad”, Revista de educación (2012), núm. 359. Madrid: Ministerio de Educación, Cultura y Deporte.

JANÉ, M., “Evaluación del aprendizaje: ¿problema o herramienta?”, Revista de estudios sociales (2005), núm. 20. Colombia: Universidad de Los Andes-Facultad de Ciencias Sociales.

MORENO OLIVOS, T., "La evaluación del aprendizaje en la universidad: Tensiones, contradicciones y desafíos”, Revista mexicana de investigación educativa (2009), núm. 41. México: Consejo Mexicano de Investigación Educativa

PIDONE, C.L., "Evaluación de los aprendizajes en la Universidad”, Diálogos educativos (2005), núm. 9. Chile: Universidad Metropolitana de Ciencias de la Educación

PORTO CURRÁS, M., "Evaluación de estudiantes en la universidad de Santiago de Compostela: percepciones de los propios alumnos", REDU: Revista de Docencia Universitaria (2009), núm. 3. Valencia: Red Estatal de Docencia Universitaria

(c) ) EY-NC-ND 2018, Universitat Politècnica de València 
QUIROGA, V., MENA GARCÍA, E., PORLAN JUY, J., MORALES MARTÍNEZ, E., MÁRQUEZ SÁNCHEZ, L., "La evaluación continua en el Grado de Trabajo Social de la Universidad de Barcelona”, Cuadernos de trabajo social (2014), núm. 2. Madrid: Universidad Complutense

RAMOS HERNANDO, C., BERASALUCE DIÉZ, R., PEIRÓ I GREGORI, S. (2016), “La evaluación por competencias en la universidad”, AAVV, XIV Jornadas de redes de investigación en docencia universitaria. Alicante: Universidad de Alicante.

SAENZ DEL BURGO MARTÍNEZ, L., PURAS OCHOA, G., “Aplicación de metodologías activas para aprender a gestionar la oficina de farmacia: Una experiencia en el aula”, IJERI: International journal of Educational Research and Innovation (2018), núm. 9. Sevilla: Universidad Pablo de Olabide.

SANTOS GUERRA, M.A., “20 paradojas de la evaluación del alumnado en la universidad española”, Revista electrónica interuniversitaria de formación del profesorado (1999), núm. 1. Zaragoza: Asociación Universitaria de Formación del Profesorado-Universidad de Zaragoza.

VIVEL BÚA, M., FERNÁNDEZ LÓPEZ, S., LADO-SESTAYO, R., "Innovación docente con One Minute Paper: ¿afecta al rendimiento académico?”, REDIE: Revista Electrónica de Investigación Educativa (2015), núm. 2. California: Universidad Autónoma de Baja California-Instituto de Investigación y Desarrollo Educativo 